\title{
Study on Causes and Control for the Transmission Difference of Urban Gas Enterprises
}

\author{
Zijian Zheng \\ Nanjing Jinsheng Gas Equipment Co., Ltd., Nanjing, China \\ Email address: \\ 1010269797@qq.com \\ To cite this article: \\ Zijian Zheng. Study on Causes and Control for the Transmission Difference of Urban Gas Enterprises. International Journal of Energy and \\ Power Engineering. Vol. 8, No. 6, 2019, pp. 94-99. doi: 10.11648/j.ijepe.20190806.14
}

Received: September 22, 2019; Accepted: December 24, 2019; Published: December 26, 2019

\begin{abstract}
Urban gas enterprises are different in management due to different regions and cultures. With the development of science and technology and the Internet, new equipment and technology are gradually combined with natural gas metering management. However, due to the incomplete development of this field in China, there is still a lot of room for progress, especially at the level of specific enterprises. At present, the metering management of many enterprises is still in the same stage At the primary stage, this paper focuses on the analysis of the causes of gas transmission difference, through observation and experiment, to find out the solutions with strong operability, and to provide valuable reference for enterprises to strengthen the management of gas transmission difference. At the beginning of this paper, the author points out the significance of the research on the measurement and transmission difference of natural gas, and then analyzes the causes of the measurement and transmission difference of natural gas through practical cases combined with historical research and experimental data. The best way is to explore the causes of the measurement and transmission difference of various types of natural gas and summarize the effective methods to reduce the measurement and transmission difference of natural gas.
\end{abstract}

Keywords: Transmission Difference, Urban Gas Enterprises, Temperature Sensor

\section{Introduction}

Because of different regions and humanities, the management methods of urban gas enterprises are also different. This paper focuses on the analysis of the reasons of natural gas transmission difference. It can not only take the case as an example, but also sort out the visiting and collecting information, and find out the solutions that can follow the rules and have strong operability. If the three major oil companies, China National Petroleum Corporation, Sinopec Group and China National Offshore Oil Corporation, are compared to the upstream (the provincial natural gas company can also be called the upstream), the urban gas enterprises belong to the downstream [1]. The gas flow between the upstream and downstream will produce a certain difference because of the distance between the pipeline network and the errors of the metering devices in the station, which we call "transmission difference". The generation of the transmission difference is inevitable. It is not difficult to reduce the transmission difference between upstream and downstream to the minimum [2]. After all, the number of meters between upstream and downstream is limited. If we analyze and investigate them one by one, we can solve the problem. But if we take the gate station of the city gas enterprise as the starting point and manage the huge number of users where the city pipeline network goes downwards, we should compare the total amount of gas used by all users with the total amount of the city gate station [3]. There are many uncertain factors in this transmission difference, which is also the concern of many city gas enterprise managers.

\section{The Significance of Analyzing Metering Transmission Difference of Natural Gas}

The Natural Gas Metering Management Department is an important functional department of urban gas enterprises, which covers from gate stations, high and medium voltage stations, industrial users, commercial users to resident users [4]. Its metering devices are of various types with uneven accuracy and repeatability. The good or bad measurement management is 
directly related to enterprise management in a large way, and in a small way, it is the vane of enterprise management. As far as the present situation is concerned, the measurement management of many enterprises is still in its infancy, the management process is not well straightened out, the management measures are monotonous or poor in pertinence, and the institution the system is not perfect [5]. Managers of urban gas enterprises should have advanced measurement management concepts so as to develop enterprises with competitive advantages and strong profitability and lay a foundation for sustainable development.

\section{Cause Analysis of the Generation of Natural Gas Transmission Difference}

"Transmission difference" is a popular word in all gas enterprises, and whether the transmission difference index is completed or not is still an index to assess the department, which leads everyone to care about how the "transmission difference" is generated and how to reduce the value of the transmission difference? Since 2009, we have paid attention to and visited some urban gas enterprises, especially Towngas joint ventures and China Resources joint ventures. At the beginning, we just cared about the appropriateness of the selection of metering devices, so as to prevent the large or small selection of metering devices from causing the natural gas flow not to be within the reasonable accuracy range of metering devices, which is considered to be one of the most basic conditions and also a prerequisite [6]. However, after 3-5 years of tracking operation, we found that we should not only focus on the selection of devices, but also devote more attention to the micro-field. In the following cases, we will analyze them one by one.

\subsection{Setting Benchmark of Metering Parameters}

China's natural gas measurement is based on the absolute atmospheric pressure of $1.01325 \mathrm{bar}$ and $20^{\circ} \mathrm{C}$. The volume obtained under the standard condition is used for trade measurement. The trade measurement and settlement are completed by volume corrector equipment. The formulas is as follows:

$\mathrm{Vn}=\mathrm{V} 0 \times \mathrm{P} 0 / \mathrm{Pn} \times \mathrm{Tn} / \mathrm{T} 0 \times \mathrm{Zn} / \mathrm{Z} 0$

Where,

Vn: Volume under standard conditions;

V0: Volume under working conditions;

Pn: Absolute pressure under working conditions;

P0: Absolute pressure under standard conditions, that is, 1.01325 bar;

Tn: Thermodynamic Temperature under working conditions, that is, $\mathrm{K}$ (273.15+actual measured temperature);

T0: Thermodynamic Temperature under standard conditions, that is, $\mathrm{K}(273.15 \pm 20)$;

$\mathrm{Zn}$ : Compression factor under standard conditions, dimensionless;

Z0: Compression factor under working conditions, dimensionless;
$\mathrm{Zn} / \mathrm{Z} 0 \approx 1$ (In urban gas metering, within 4 bar of absolute pressure).

In the above formulas, $\mathrm{P} 0$ and $\mathrm{T} 0$ are the benchmark values. In some countries or regions, the benchmark values of temperature are set according to $15^{\circ} \mathrm{C}$, i.e. $273.15+15$; in others, according to $0^{\circ} \mathrm{C}$, i.e. $273.15+0$; and the error is about $1 \%$ for every increase or decrease of temperature by $3.3^{\circ} \mathrm{C}$. The benchmark value of absolute pressure is based on 1.01325bar. When the volume corrector is put into use, it needs to be calibrated by a third party to confirm whether it conforms to the local metrology regulations. The temperature and pressure sensors are calibrated during calibration, and flow pulse acquisition is checked [7]. We once found that a gas company purchased imported metering devices in Zhejiang. When the volume corrector left the factory, its parameters were set according to German metering standards. The standard temperature was set to $0^{\circ} \mathrm{C}(273.15 \mathrm{~K})$. Therefore, in the settlement of trade, the measurement was reduced by about $6 \%$. The reason is that the temperature sensor is all right when metering, but there is a problem when converting the working flow into standard flow. This is true in Germany, but in China, it is necessary to set the standard values as the benchmark parameters stipulated in GB/T 21391-2008 Gas Turbine Flow Meter for Natural Gas Flow Measurement, the Chinese metrology regulation [8]. The pressure benchmark values are implemented according to $1.01325 \mathrm{bar}$, and the implementation standards are the same in different countries or regions.

\subsection{Metering Devices Affected by Environmental Temperature}

In the process design stage, the designer did not fully consider the installation environment of the metering devices. When the devices were installed and put into operation, it was found that the environmental temperature had a greater impact on the metering [9]. The most prominent case is the installation of metering device in the basement boiler room of a hospital (see Figures 1, 2).

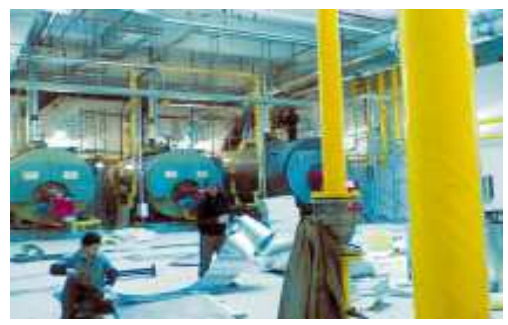

Figure 1. Basement Boiler Room.

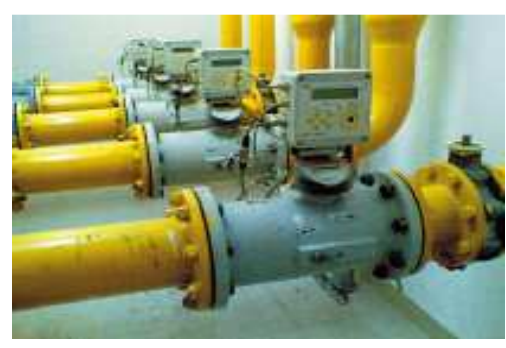

Figure 2. Basement Equipment Room. 
Affected by the heat source of the adjacent boiler, the average indoor temperature is $37^{\circ} \mathrm{C}$ in summer, $31^{\circ} \mathrm{C}$ in winter and $32^{\circ} \mathrm{C}$ to $34^{\circ} \mathrm{C}$ in annual, while the actual temperature of natural gas in the pipeline is $2^{\circ} \mathrm{C}$ to $3^{\circ} \mathrm{C}$ lower than the metered value. Why does this happen? After on-site inspection, communication with relevant personnel of the owner, and understanding of the gas consumption of the boiler operation, the following points were found:

(1) The natural gas intake pipe enters the basement and distributes pipes along the boiler and wall. The distance is about 1 meter. There are three boilers in one row and two boilers on the other side. The length of each pipeline installed in the basement is about 30 meters. Each boiler corresponds to a turbine flowmeter;
(2) The equipment room of flowmeter is located above the boiler, which is the place with the highest temperature in the whole basement;

(3) The air flow in the basement can not disperse and the ambient temperature is difficult to decrease;

(4) There are five boilers in winter, at least three boilers were operated and one to two boilers were operated in summer;

(5) The temperature variation of natural gas metered by the flowmeter is relatively small, with only $0.2-0.3^{\circ} \mathrm{C}$ fluctuation in four hours.

For this user, we randomly sampled the data of one of the flowmeters and recorded the data in Table 1 below.

Table 1. Sample flowmeter data.

\begin{tabular}{llllll}
\hline Time & Type of Flowmeter & Measured Temperature & $\begin{array}{l}\text { Ambient Temperature of } \\
\text { Equipment Room }\end{array}$ & $\begin{array}{l}\text { Local Ambient } \\
\text { Temperature }\end{array}$ & $\begin{array}{l}\text { Gas } \\
\text { Flow }\end{array}$ \\
\hline Jul. 21 & TRZ2G400DN150/EK260 & $38.95^{\circ} \mathrm{C}$ & $39^{\circ} \mathrm{C}$ & $36^{\circ} \mathrm{C}$ & $212 \mathrm{~m}^{3} / \mathrm{h}$ \\
\hline
\end{tabular}

From the data in the table, it can be seen that the ambient temperature of the equipment room directly affects the measuring temperature of the volume corrector, and the temperatures are almost the same. The natural gas pipeline, flowmeter surface and temperature sleeve are all metals with good thermal conductivity [10]. External temperature directly acts on the metal and transfers to temperature sensor. Under the condition of low natural gas flow rate, the actual temperature of natural gas can not be measured (see Figure 3).

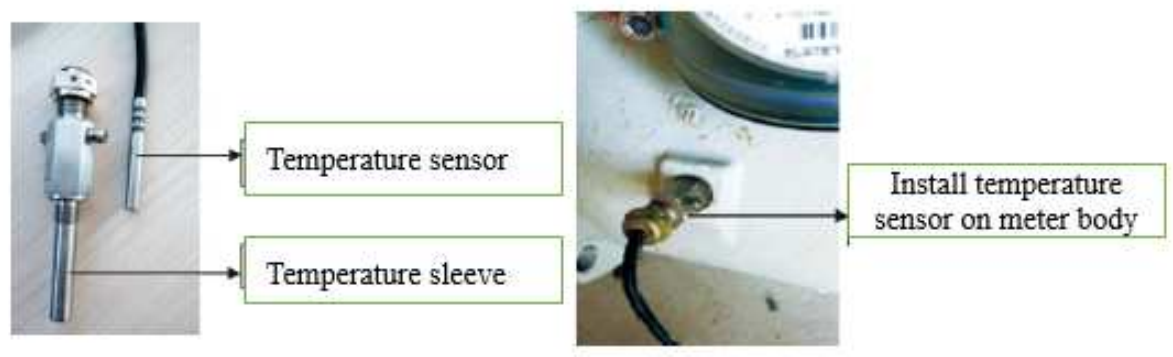

Figure 3. Installation of Temperature Sensor.

In this special environment, the existing temperature measurement method has been improved, that is, the temperature sensor directly measures the temperature of natural gas (the improved temperature sensor is suitable for less than 2bar pressure), so that the influence of external interference will be reduced (see Figure 4).
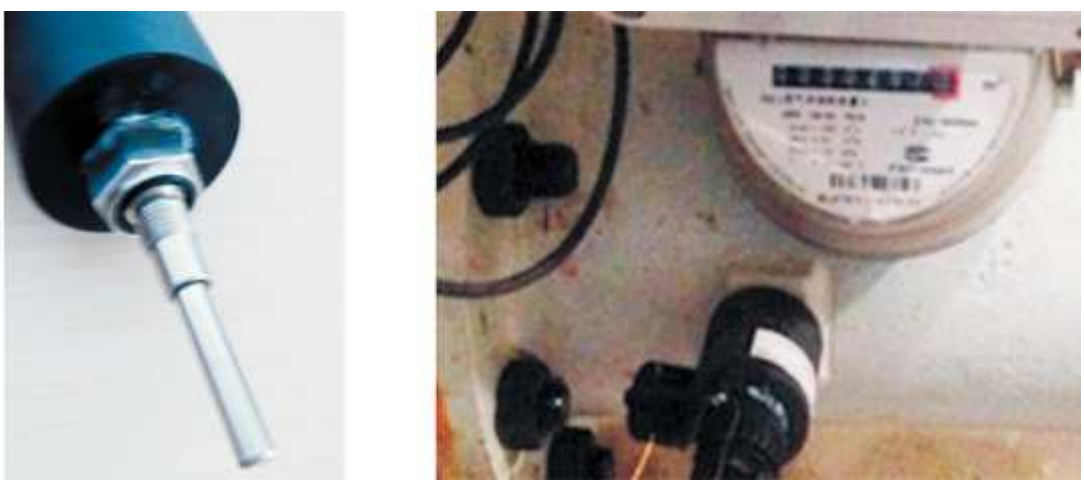

Figure 4. Installation Diagram of the Improved Temperature Sensor.

The improved temperature sensor was installed on the flowmeter of the hospital and the data as shown in Table 2 were obtained. 
Table 2. Sample flowmeter data.

\begin{tabular}{llllll}
\hline Time & Type of Flowmeter & Measured Temperature & $\begin{array}{l}\text { Ambient Temperature of } \\
\text { Equipment Room }\end{array}$ & Local Ambient Temperature & Gas Flow \\
\hline Jul. 23 & TRZ2G400DN150/EK260 & $36.95^{\circ} \mathrm{C}$ & $39.3^{\circ} \mathrm{C}$ & $37^{\circ} \mathrm{C}$ & $287 \mathrm{~m}^{3} / \mathrm{h}$ \\
\hline
\end{tabular}

The data show that the temperature of natural gas is about $2.35^{\circ} \mathrm{C}$ lower than the ambient temperature, and the transmission difference is reduced by about $0.7 \%$.

\subsection{Pipeline Impurity}

After the new user turns on the natural gas and the flowmeter is put into normal operation, the differential pressure gauge on the front end filter is generally observed in the first 3 months to prevent the impurity from sealing the filter element. The optional filter element has a filtration accuracy of not less than 50um. Some users choose the filter element with 100 um filtering accuracy when installing the filter unit, in order to prevent the filter element from being blocked and frequently replacing the filter element. In most pipeline construction process, it is not carried out in accordance with the norms and operating procedures [11]. The inadequate cleaning of the pipeline results in various impurities in the pipeline: welding slag, sediment, water, and even electrodes, gloves and safety caps. These impurities have great hidden dangers to the accurate measurement of flowmeters, and some of them have directly harmed the flowmeters [12]. When a large object enters the filter, it will block one side of the filter element and produce disturbed flow, which will affect the metering; when water enters the surface, it will corrode the measuring core bearing and rotating parts, which will lead to an increase of flow rate when the flowmeter starts; when welding slag or sediment enters the surface, it will directly damage the turbine or lumbar wheel rotor, or even block the blade or rotor, resulting in the failure of operation (see Figure 5).
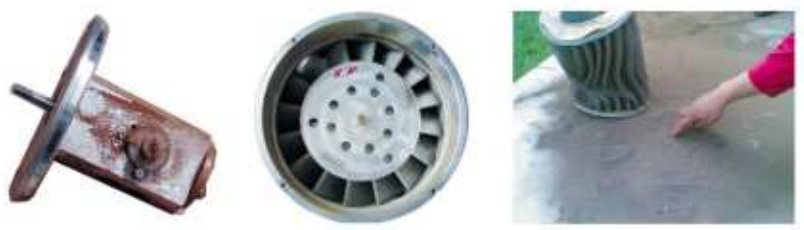

Figure 5. Impurity in the Measuring Element.

Because the filter element is blocked by impurities, the pressure difference is large, leading to the deformation of the filter element and thus resulting in a large number of fine particles into the sensor and attached to the precision measuring core gear. The flowmeter shown above takes no more than three months from being put into operation to problems [13]. During the three-month operation period, the flowmeter is measured in a relatively harsh environment, the accuracy is less than $1 \%$, and the flow curve is in a negative deviation, which is contrary to the linearity of factory or calibration. The linearity of a general turbine flowmeter is shown in Figure 6.

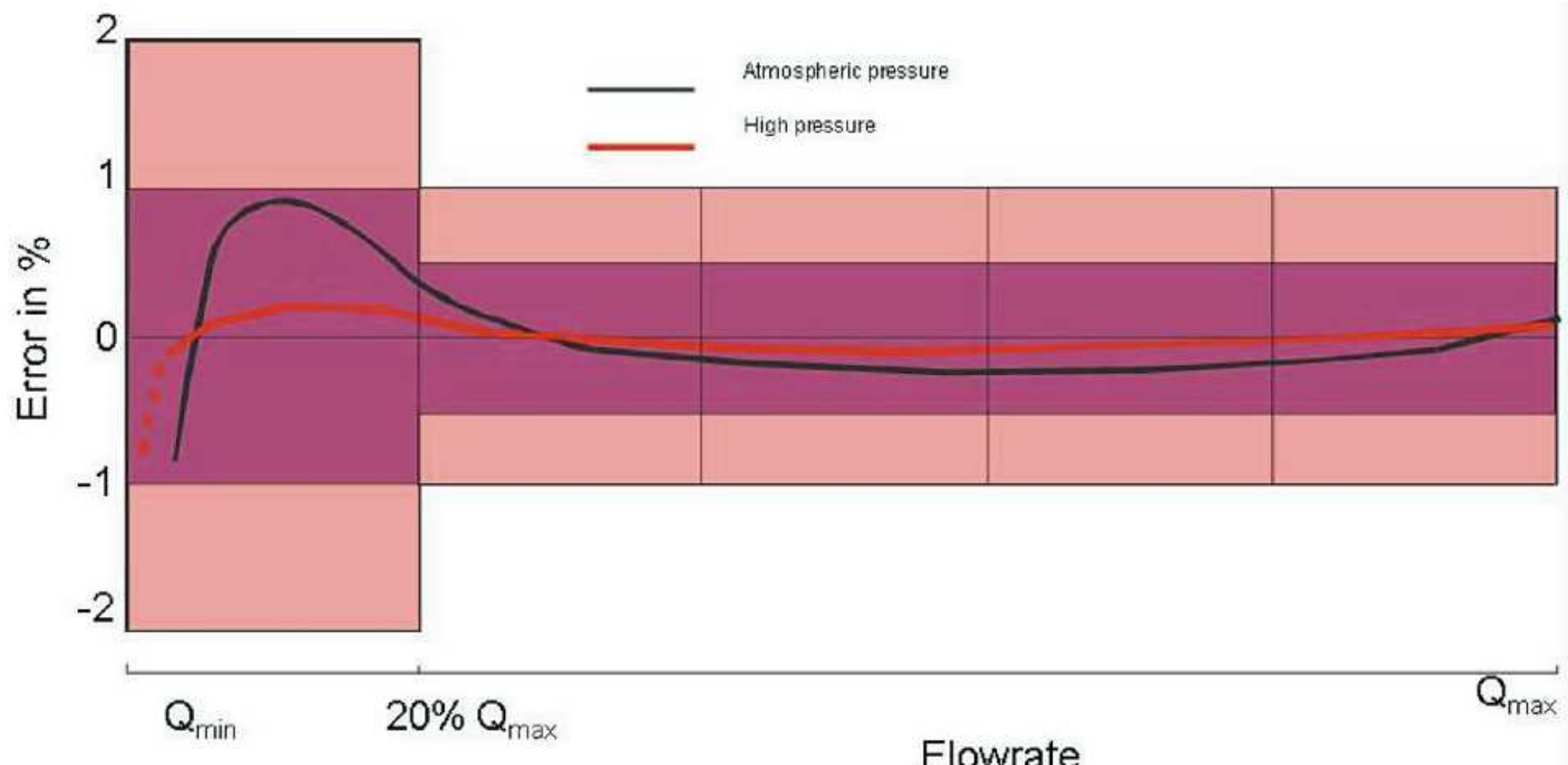

Figure 6. Linearity of Turbine Flowmeter.

\subsection{Misoperation}

Flowmeters need special personnel to manage them. They need to understand the performance and principle of flowmeters, so that they will not operate blindly. Taking the turbine flowmeter as an example, when operating and managing the turbine flowmeter, the function of the 
flowmeter and the characteristics of its auxiliary components should be well mastered [14]. As shown in Figure 6, it is divided into two parts: the base meter of turbine flowmeter and the volume corrector. The two parts are connected by pressure tube, temperature sensor and pulse, and some flowmeters are equipped with oil pump. The connection between temperature sensor and pulse is basically unmovable. The pressure-relief valve connected by pressure sensor should be open regularly. The small ball valve should be closed only when the volume corrector is dismantled. In many metering disputes, when the pressure diversion valve is not opened, the ventilation metering is carried out [15]. After two or three months or six months of operation, it is found that the small ball valve is not opened and the volume corrector does not compensate the pressure of natural gas in the metering. When operating under pressure of less than lbar, the pressure of more or less $1 \mathrm{kPa}$ will increase or decrease the transmission difference by $1 \%$.

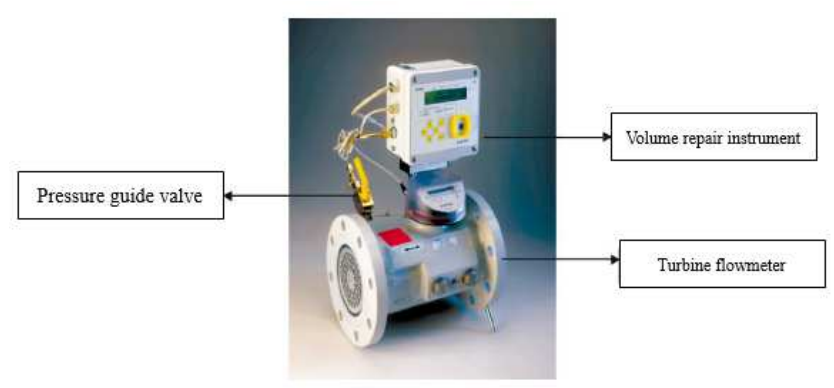

Figure 7. Installation Diagram of Turbine Flowmeter and Volume Repair Instrument.

For flowmeters equipped with pumps, users seldom refuel the flowmeters every 2-3 months. They refuel intentionally or unintentionally, regardless of whether the flowmeters are running or not. We know that the function of the oil pump of the flowmeter is to wash away the impurities in the measuring core through the high-pressure lubricant when the flowmeter is running, and the impurities and oil flow downstream along the natural gas. It is impossible to refuel the flowmeter when it is in a stationary state. If the lubricating oil in the oil pump has not been used up for more than a year or the color becomes darker, it should be removed and replaced with new special lubricating oil.

\subsection{Other Reasons}

There are also some factors that cause the transmission difference of natural gas metering, which are more common and concerned by all of us. For example, for the accuracy level of flowmeter selection, the $\pm 1 \%$ accuracy with high cost performance is generally selected, and the mechanical head is equipped with volume corrector. The mechanical head is the original data, which is not easy to generate trade disputes, and easy to calculate the operation status of the flowmeter. In the process design, it is necessary to satisfy the straight pipe section (straight pipe section with the drift diameter not less than 2DN) with long enough inlet of turbine flowmeter, so that the flow pattern is stable and the measurement is accurate [16]. There is also the phenomenon of man-made destruction of metering equipment, more of which are made by residential users and small industrial and commercial users, in order to achieve metering equipment without metering or less metering, etc. There is no more elaboration here.

\section{Conclusion}

Based on the analysis of the causes of gas transmission difference, through the observation and experiment of examples, this paper obtains the solutions with strong operability, which provides valuable reference for enterprises to strengthen the management of gas transmission difference. The details are as follows:

The flowmeter is managed by specially-assigned person who are fixed professionals. For the specially-assigned person, the emphasis is on the quality rather than the number. Generally, two people need to go to the scene to check the equipment. For some large gas users, first of all, they need to understand the characteristics of the gas equipment, and keep track of the gas consumption at any time.

Strict implementation of metering laws and regulations. The first inspection and periodic verification of flowmeters should be strictly carried out. Turbine flowmeters are inspected once every eight years in Europe and not more than two years in China. As mentioned above, there are various impurities in the gas pipelines of some cities. The flowmeters are damaged less than three months after installation, and some of them are working with damages. Therefore, periodic verification should be arranged reasonably according to the existing pipeline conditions of the company. During the periodic verification, not only the base meter of flowmeter should be inspected, but also the volume corrector should be calibrated to prevent the pressure or temperature sensor from drifting. The periodic verification time of base meter can be one year or two years. If there are many impurities in the pipeline, the meter can be removed every six months to clean up the filter device to ensure that the flowmeter is measured safely, stably and accurately.

Strengthen the management of the seal. For the key components which can affect the metering, the specially-assigned person shall seal the metering devices, register the number of the seal, and mark markedly on the flowmeter, so as to prohibit the destruction of the seal.

\section{References}

[1] Liang Xiang. Discussions on the relevant measures to implement the differential management in urban gas management [J]. Low Carbon World, 2019, 9 (04): 123-124.

[2] Jingru Liu. Causes and control of poor natural gas transmission [J]. Chemical design communication, 2019, 45 (08): $54+56$.

[3] Qingjuan Guo. Measures to strengthen the management of gas transmission difference in pipelines [J]. Modern economic information, 2019 (10): 390. 
[4] Bin Guo. Research on the Construction of Urban Gas Transmission and Distribution Pipeline Network [J]. Scientific and Technological Innovation and Application, 2019 (07): 86-87.

[5] Dongsheng Peng. Safety Management of Urban Gas Transmission and Distribution System [J]. Science and Technology Economic Guide, 2018, 26 (15): 234.

[6] $\mathrm{Xu}$ Zhao. Talking about how to strengthen the dispatching management of urban gas transmission and distribution [J]. Sichuan Cement, 2017 (12): 197.

[7] Hui Xiang. Cause analysis and Countermeasures of gas meter metering transmission difference $[\mathrm{J}]$. Science and technology innovation, 2019 (14): 174-175.

[8] Yuan Gao. Application of Intelligent Technology in Urban Gas Transmission and Distribution Pipeline System [D]. Beijing University of Architecture, 2017.

[9] Yancong Liu. Discussion on Some Problems of Urban Gas Transmission and Distribution Pipeline Network [J]. Shanxi Science and Technology, 2017, 33 (03): 123-125.

[10] Feng Cheng. Analysis and Management of Urban Gas Transmission Difference [J]. Urban Gas, 2016 (07): 40-43.
[11] Guoping Gu. Problems and Countermeasures of gas transmission gap $[\mathrm{J}]$. Scientific and technological innovation and application, 2015 (23): 150.

[12] Jing Zhang. Analysis of the causes and control points of gas transmission difference $[\mathrm{J}]$. Research on modern state-owned enterprises, 2018 (12): $129+41$.

[13] Jin $\mathrm{Xu}$. Problems and control analysis of urban gas transmission gap [J]. China Management Informatization, 2015, 18 (14): 103.

[14] Shiyi Yang. Research on metering and transmission difference control of urban gas pipeline network [D]. Southwest Petroleum University, 2015.

[15] Saipeng Qi. Brief analysis of formation factors and control strategies of urban gas transmission gap [J]. Chemical Industry Management, 2014 (08): 150.

[16] Lisa Liao. Measures for Implementing Transport Difference Management in Urban Gas Management [J]. Enterprise Reform and Management, 2016 (18): 209. 